\title{
A Numerical Methodology to Fully Elucidate the Altered Wall Shear Stress in a Stented Coronary Artery
}

Jonathan Murphy

Technological University Dublin, jonathan.murphy@tubublin.ie

Fergal Boyle

Technological University Dublin, fergal.boyle@tudublin.ie

Follow this and additional works at: https://arrow.tudublin.ie/engschmecart

Part of the Computer Engineering Commons

\section{Recommended Citation}

Murphy, J., Boyle, F.: A Numerical Methodology to Fully Elucidate the Altered Wall Shear Stress in a Stented Coronary Artery. Cardiovascular Engineering and Technology,Vol. 1, Number 4, pp256-268. 2010. doi:10.1007/s13239-010-0028-0

This Article is brought to you for free and open access by the School of Mechanical and Design Engineering at ARROW@TU Dublin. It has been accepted for inclusion in Articles by an authorized administrator of ARROW@TU Dublin. For more information, please contact arrow.admin@tudublin.ie, aisling.coyne@tudublin.ie, gerard.connolly@tudublin.ie.

Funder: Department of Mechanical Engineering, Technological University Dublin (DIT)and the Irish Research Council for Science Engineering and Technology (IRCSET) 


\title{
A Numerical Methodology to Fully Elucidate the Altered Wall Shear Stress in a Stented Coronary Artery
}

\author{
Jonathan B. Murphy and Fergal J. Boyle \\ Department of Mechanical Engineering, Dublin Institute of Technology, Bolton Street, Dublin 1, Ireland
}

(Received 29 May 2010; accepted 26 October 2010)

Associate Editor John M. Tarbell oversaw the review of this article.

\begin{abstract}
Arterial restenosis after coronary stenting is caused by excessive tissue growth which is stimulated by arterial injury and alterations to the hemodynamic wall shear stress (WSS). Recent numerical studies have predicted only minor differences in the altered WSS between different stent designs using a commonly employed threshold assessment technique. While it is possible that there are only minor differences, it is more likely that this assessment technique is incapable of fully elucidating the alterations to the WSS created by stent implantation. This paper proposes a methodology that involves a more complete investigation into the stent-induced alterations of WSS by incorporating the full suite of WSS-based variables: WSS, WSS gradient (WSSG), WSS angle gradient (WSSAG) and oscillatory shear index (OSI). The four variables are analyzed quantitatively and qualitatively to assess the effect of the stent implantation. The methodology is applied to three stents with contrasting designs: the Palmaz Schatz (PS), Gianturco Roubin II (GR-II) and Bx-Velocity (Bx) stents. For WSS the methodology ranks the stents (best to worst) as follows: PS, GR-II, Bx (Cohen's $d$ : $-0.01,-0.613)$, for WSSG: PS, Bx, GR-II $(d: 0.159,0.764)$, for WSSAG: PS GR-II Bx (d: 0.213, $0.082)$, and for OSI: PS, GR-II, Bx (d: 0.315, 0.380). The suggested quantitative and qualitative assessment of the WSS-based variables is shown to improve upon, and highlight the weakness of, the previously used threshold assessment technique. The proposed methodology could be utilized to minimize WSS alterations at the design stage of future coronary stents.
\end{abstract}

Keywords-Pulsatile flow, Restenosis, Multi-variable analysis.

\section{INTRODUCTION}

Balloon angioplasty is a minimally invasive interventional technique to restore blood flow through coronary arteries constricted by atherosclerosis.

Address correspondence to Jonathan B. Murphy, Department of Mechanical Engineering, Dublin Institute of Technology, Bolton Street, Dublin 1, Ireland. Electronic mail: jonathan.murphy@dit.ie
However, a subsequent re-blockage or restenosis can occur if the artery elastically recoils back to its narrowed state. Bare metal stents (BMSs) were introduced to prevent this arterial recoil but restenosis remains a significant problem with BMSs occurring in between 10 and $50 \%$ of patients treated. ${ }^{12,17}$ Restenosis in stented arteries is essentially caused by the excessive growth of new tissue in the stented segment of the artery, a process termed intimal hyperplasia (IH). Currently, the implantation of drug-eluting stents (DESs) has proven effective in reducing restenosis rates to below $10 \%$. $^{8,31}$

In a clinical study, ${ }^{17}$ stent design was found to be the second strongest risk factor, after vessel size, for restenosis at six-month follow-up angiography among 3370 patients. Stent-design-related stimuli for restenosis are arterial injury and altered hemodynamics. The influence of stent design on arterial injury has been demonstrated ${ }^{38}$ and the severity of the injury correlated to the volume of subsequent tissue growth. ${ }^{38,39}$ Altering the hemodynamic wall shear stress (WSS) at the artery wall through stent implantation has also been shown to influence arterial tissue growth. ${ }^{14,26}$ Previous works ${ }^{5,22,30}$ which have linked altered hemodynamics to stent design prompted further investigations into the hemodynamic environment of the stented artery.

Currently, state-of-the-art numerical investigations into WSS in the stented artery employ threedimensional (3D) and transient computational fluid dynamics (CFD). ${ }^{3,4,6,10,11,15,21,23,25,37}$ In these studies the alteration of the WSS acting on the wall is sometimes analyzed using threshold values of the WSS (WSS $<0.5 \mathrm{~N} / \mathrm{m}^{2}$ ) and WSS gradient (WSSG $>$ $200 \mathrm{~N} / \mathrm{m}^{3}$ ) as tissue growth has been found to be more prolific in areas where the WSS and WSSG are lower and higher than these thresholds, respectively. ${ }^{18,20}$ One such study ${ }^{23}$ examined the effect of stent foreshortening 
on WSS, and found that for 0,12 , and $25 \%$ foreshortening there was 71,77 , and $78 \%$ of the stented area with WSS $<0.5 \mathrm{~N} / \mathrm{m}^{2}$ and 54,50 , and $54 \%$ of the stented area with $\mathrm{WSSG}>200 \mathrm{~N} / \mathrm{m}^{3}$, respectively. In a more recent study, ${ }^{3} \mathrm{CFD}$ analyzes of four commercially available BMSs were conducted. Comparisons between two of the stents with contrasting design, the Bx-Velocity stent (Cordis Corp., Miami, FL, USA) and the Jostent Flex (JOMED AB, Helsingborg, Sweden), showed 58 and $57 \%$ of the stented area with WSS $<0.5 \mathrm{~N} / \mathrm{m}^{2}$ respectively. The effect of strut thickness on WSS was also examined using Jostent Flex stents with strut thicknesses of 0.05 and $0.15 \mathrm{~mm}$. The thinner struts led to $61 \%$ of the stented area with WSS $<0.5 \mathrm{~N} / \mathrm{m}^{2}$ compared with $57 \%$ for the thicker struts. Duraiswamy et al. ${ }^{11}$ also conducted CFD analyzes of three, second-generation, commercially available BMSs: the Bx-Velocity stent, the Aurora stent (Medtronic, Inc., Minneapolis, MN, USA) and the NIR stent (Boston-Scientific Corp., Natick, MA, USA). They found 59, 57, and $59 \%$ of the stented area with WSS $<0.5 \mathrm{~N} / \mathrm{m}^{2}$ and 75,83 , and $88 \%$ of the stented area with WSSG $>200 \mathrm{~N} / \mathrm{m}^{3}$ for the Bx-Velocity, Aurora and NIR stents, respectively.

These previous studies depict only minor differences in the altered WSS between stent designs. However, numerous studies have shown that stent strut angle, ${ }^{26}$ strut thickness ${ }^{16,35}$ and strut configuration ${ }^{17,27,38}$ have a significant influence on the arterial response to stent implantation. In light of this, it is highly probable that using threshold values of only the WSS and WSSG variables is not sufficient to fully elucidate stentinduced alterations in arterial WSS.

In this work, a numerical prediction methodology is proposed which utilizes the two commonly used variables (i.e., WSS and WSSG) plus two additional WSSbased variables, the WSS angle gradient (WSSAG) and the oscillatory shear index (OSI). Each of these variables highlights a different type of alteration to the arterial WSS which could lead to IH. Instead of using threshold values, statistical measures of the full distribution of each of these variables are calculated for a more complete analysis. To demonstrate the methodology, it is applied to three stents with contrasting features. The stents are then ranked based on the statistical measures. Comparisons are made between the results from the analyzes and the two-variable threshold analysis described above. The proposed methodology can be used to identify areas of altered arterial WSS at the design stage of future bare metal, as well as permanent and bioabsorbable drug-eluting coronary stents.

The paper is laid out as follows: "Materials and Methods" section describes the proposed methodology which includes the generation of the computational domain, the CFD analysis and the post-processing. The results from the application of the methodology to three stents are presented in "Results" section, with the conclusions given in "Discussion" section.

\section{MATERIALS AND METHODS}

The methodology begins with the generation of a geometric model of the 3D lumen of the stented artery. This model is discretized and used as the computational domain for the CFD analysis. The WSS vectors are predicted on domain surfaces that represent living tissue. These vectors are then post-processed to produce the magnitudes of the four WSS-based variables: WSS, WSSG, WSSAG and OSI. The distribution of these variables is then analyzed using quantitative and qualitative statistical methods.

\section{Generation of the Computational Domain}

A 3D geometric model is first created beginning with a solid cylinder measuring the length and external diameter of the stent, from which the geometry of the complete stent is removed. This represents full strut exposure to the blood flow. The geometric model is then extended proximal and distal to the stented section by adding cylinders with lengths equal to the entrance length for fully-developed laminar flow in a circular pipe and with diameters which create a stentto-artery deployment ratio of 1.09:1 similar to a normal in vivo value. ${ }^{27}$ This ratio is defined as the inner diameter of the deployed stent to the inner diameter of the unstented artery. Finally, a novel methodology to numerically predict tissue prolapse between stent struts $^{32}$ is employed. Briefly, the prolapsing tissue creates a variable arterial radius $r$ given by

$$
\frac{r}{R_{0}}=1-\frac{x \delta}{2 R_{0}}\left\{1+\cos \frac{2 \pi}{L}\left(z-\frac{L}{2}\right)\right\}
$$

along the spatial coordinate $z$ between two stent struts, where $R_{0}$ is the external diameter of the stent, $L$ is the distance between the stent struts, $\delta$ is the prolapse depth and $\delta=C L$ where $C$ is the coefficient of prolapse derived from finite element analysis (FEA) data. ${ }^{36}$ The prolapse reduction factor $x$ is initiated at a distance of $0.5 \mathrm{~L}$ from struts offering additional scaffolding support as shown in Fig. 1. The prolapse reduction factor linearly decreases the prolapse to zero at the stent strut providing the additional support. The prolapsing tissue is then removed from the geometric model. The geometric model thus created is discretized using an unstructured mesh topology comprising of 4-node tetrahedral elements to form the computational domain. Several simulations are performed to 
investigate the effect of mesh density to ensure meshindependent results are obtained. This methodology is applied to stents closely resembling the Palmaz Schatz (PS) stent (Johnson and Johnson Interventional System, Warren, NJ, USA), the Gianturco Roubin II (GR-II) stent (Cook Inc., Bloomington, IN, USA) and the Cordis Bx-Velocity (Bx) stent (Johnson and Johnson Interventional System, Warren, NJ, USA) all implanted in the left-anterior-descending (LAD) coronary artery (Fig. 2). These contrasting stent designs are chosen specifically to identify the effects of the different geometric features on the WSS. Also, the prior knowledge of the in vivo performance of these stents is useful when analyzing the results as the altered hemodynamics may have contributed to their restenosis rates.

Details of the 3D geometric models are provided in Table 1. The unstented sections of the artery are $3.2 \mathrm{~mm}$ in diameter and $21.3 \mathrm{~mm}$ in length. Meshindependent results are achieved with 4,551,484 elements for the PS stent, 3,038,536 elements for the GR-II stent and 5,840,890 elements for the Bx stent.

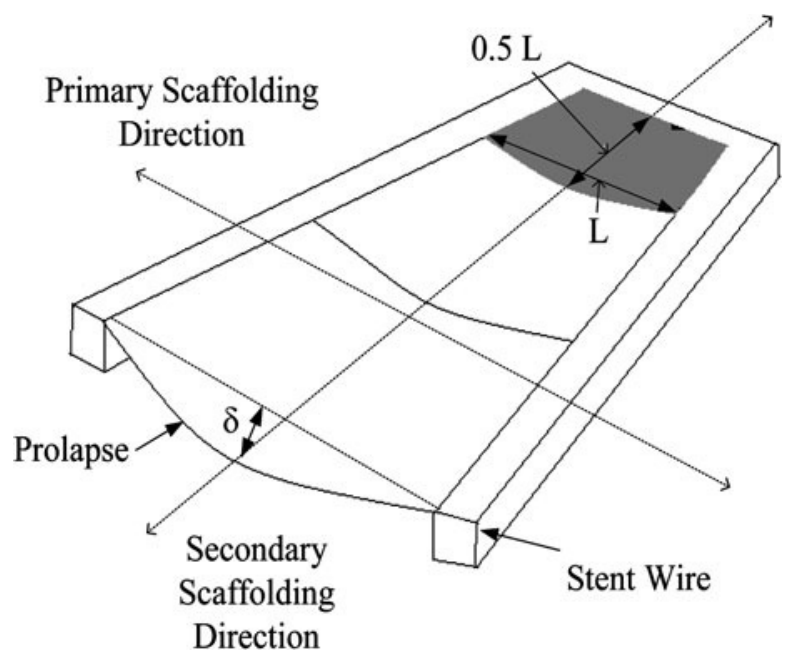

FIGURE 1. Illustration of the primary and secondary scaffolding direction of part of the PS stent. The arterial tissue protrudes a depth $\delta$ into the stented artery and is supported in the primary scaffolding direction and partially supported (shaded section) in the secondary scaffolding direction.
On the stent struts, the meshes have a maximum element edge length of $20-30 \mu \mathrm{m}$ depending on the stent and a minimum edge length of $1 \mu \mathrm{m}$ for all stents to allow adequate resolution of any complex geometric features. Elements in the center of the artery have a maximum edge length of $200 \mu \mathrm{m}$. Mesh independence is based on a less than $4 \%$ change in the RMS value of the magnitudes of WSS vectors between successive meshes along sample lines in the domain, an example of which is shown in Fig. 3a.

\section{CFD Analysis}

The general form of the governing flow equations for the conservation of mass and momentum are given in vector form in Eqs. (2) and (3), respectively:

$$
\begin{gathered}
\frac{\partial \rho}{\partial t}+\vec{\nabla} \cdot(\rho \vec{V})=0 \\
\frac{\partial \rho \vec{V}}{\partial t}+\vec{\nabla} \cdot(\rho \vec{V} \otimes \vec{V})=-\vec{\nabla} p+\vec{\nabla} \cdot\left(\overrightarrow{\vec{\tau}}_{i j}\right)
\end{gathered}
$$

where $\rho$ is the fluid density, $p$ is the static pressure and $\vec{V}$ is the velocity vector. In this work, the blood flow in

TABLE 1. Geometric characteristics of the stent models.

\begin{tabular}{llll}
\hline Geometric characteristic & Palmaz Schatz & Gianturco Roubin II & Bx-Velocity \\
\hline Length $(\mathrm{mm})$ & 14.3 & 20.0 & 12.5 \\
Internal diameter $(\mathrm{mm})$ & 3.5 & 3.5 & 3.5 \\
Strut thickness $\times$ width $(\mathrm{mm})$ & $0.0635 \times 0.152$ & $0.076 \times 0.172$ & $0.14 \times 0.13$ \\
Stent total surface area $\left(\mathrm{mm}^{2}\right)$ & 108.92 & 83.84 & 100.31 \\
Prolapsing tissue volume $\left(\mathrm{mm}^{3}\right)$ & 5.71 & 15.12 & 6.52 \\
Normalized prolapsing tissue volume $\left(\mathrm{mm}^{3} / \mathrm{mm}\right)$ & 0.40 & 0.76 & 0.52 \\
Stent to tissue contact area $\left(\mathrm{mm}^{2}\right)$ & 44.71 & 30.91 & 23.69 \\
Normalized stent to tissue contact area $\left(\mathrm{mm}^{2} / \mathrm{mm}\right)$ & 3.13 & 1.55 & 1.90 \\
\hline
\end{tabular}

Certain values are normalized by the stent length. 

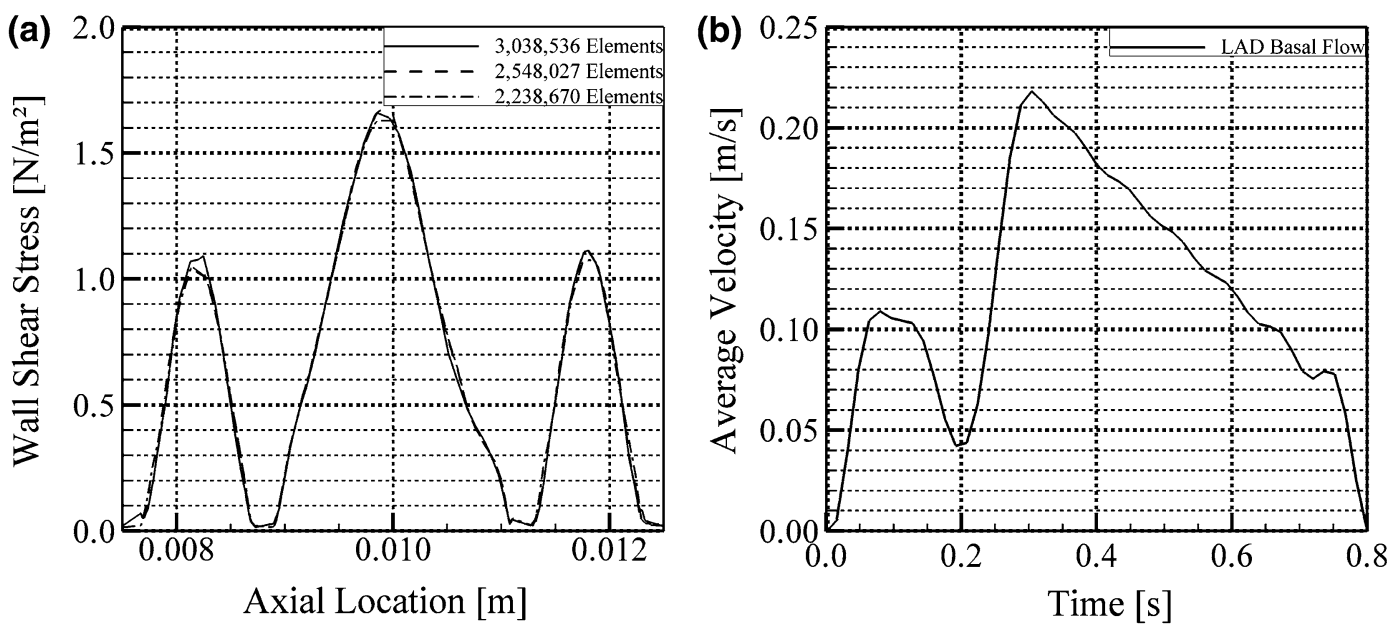

FIGURE 3. (a) Magnitude of WSS along a sample line in the model of the LAD coronary artery implanted with a GR-II stent for three successive mesh densities and (b) average velocity applied at the inlet to simulate basal flow in the LAD coronary artery.

the stented artery is assumed time-dependent, laminar and incompressible. With these assumptions the viscous stress tensor $\overrightarrow{\vec{\tau}}_{i j}$ is given by

$$
\overrightarrow{\vec{\tau}}_{i j}=\mu\left(\vec{\nabla} \vec{V}+\vec{\nabla} \vec{V}^{T}\right)
$$

where $\mu$ is the fluid dynamic viscosity, which is a function of the shear rate. The governing equations are solved by the commercial software package ANSYS CFX Version 12 (Canonsburg, PA, USA) in a Cartesian coordinate system using a vertex-centered finitevolume scheme with implicit time-stepping, a scheme which is second-order accurate in both space and time.

A transient, fully-developed, laminar, axial-velocityprofile is applied at the inlet of the computational domain which corresponds to the desired blood flow conditions in the coronary artery. A fixed static pressure equal to the reference pressure of $114600 \mathrm{~Pa}$ is applied at the outlet of the domain. This pressure represents the mean arterial blood pressure in the LAD coronary artery. The no-slip boundary condition is applied on all surfaces representative of the artery wall and the stent struts. The blood density is assumed constant with a value of $1050 \mathrm{~kg} / \mathrm{m}^{3}$. The nonNewtonian nature of the flow is accommodated using the Carreau model written

$$
\mu=\mu_{\infty}+\left(\mu_{0}-\mu_{\infty}\right)\left[1+(\gamma \lambda)^{2}\right]^{\left(\frac{q-1}{2}\right)}
$$

where $\gamma$ is the shear rate calculated as the second invariant of the strain rate tensor and the constants are

$$
\begin{array}{rr}
\mu_{0}=0.056 \mathrm{Ns} / \mathrm{m}^{2} & \lambda=3.31 \mathrm{~s} \\
\mu_{\infty}=0.00345 \mathrm{Ns} / \mathrm{m}^{2} & q=0.375
\end{array}
$$

At each timestep the convergence criterion employed is a $10^{-5}$ reduction in the maximum residuals of the discretized equations. Simulations are run for three cardiac cycles to ensure periodic convergence of the results. Several simulations also are conducted to investigate the effect of the timestep so as to ensure temporal convergence is achieved.

The flow rate chosen corresponds to fully-developed pulsatile basal flow conditions in the human LAD coronary artery ${ }^{33}$ and is shown in Fig. 3b. The average Reynolds number for the cycle is 111 and the average flow rate is $55 \mathrm{~mL} / \mathrm{min}$. The period of the cardiac cycle is $0.8 \mathrm{~s}$, and the simulation is run for three consecutive cycles with a timestep of $12.5 \mathrm{~ms}$. This timestep is sufficient to ensure temporal convergence is achieved. Computations are performed on a HP xw6400 64-bit workstation using two processors of a quad Intel (Xeon) $2 \mathrm{GHz}$ CPU and $6 \mathrm{~GB}$ of RAM.

\section{Post-Processing}

The vertex-centered finite-volume scheme employed by ANSYS CFX calculates the variables at the vertices of the elements. All post-processing to calculate the WSS-based variables is then conducted in Tecplot 360 2008 (Bellevue, WA, USA). The WSS, WSSG, and components of the WSSAG and OSI are calculated at each timestep of the third cycle and are then timeaveraged over this cycle using the trapezoidal method of numerical integration. The calculation of these variables is described below.

\section{Wall Shear Stress}

Lower than physiologic values of WSS $\left(<1.5 \mathrm{~N} / \mathrm{m}^{2}\right)$ can cause dysfunction of the endothelial cells (ECs) which line the artery. Under such conditions, ECs act as a catalyst for IH through, for example, the 
upregulation of tissue growth factors such as PDGF-A and PDGF-B. ${ }^{29,45}$ Numerous studies which incorporate numerical and experimental results have correlated areas of low WSS with increased $\mathrm{IH}^{26,43,46,47}$

The dot product of the unit normal vector $\vec{l}$ to a surface and the viscous stress tensor denoted $\overrightarrow{\vec{\tau}}_{i j}$ yields the WSS, i.e.,

$$
\vec{l} \cdot \overrightarrow{\vec{\tau}}_{i j}=\vec{\tau}_{w_{x y z}}=\tau_{w, x} \vec{i}+\tau_{w, y} \vec{j}+\tau_{w, z} \vec{k}
$$

where $\tau_{w, x}, \tau_{w, y}$ and $\tau_{w, z}$ are the Cartesian components of the WSS vector in the $x, y$ and $z$ directions, respectively. The magnitude of the WSS vector is calculated as

$$
\mathrm{WSS}=\left(\tau_{w, x}^{2}+\tau_{w, y}^{2}+\tau_{w, z}^{2}\right)^{1 / 2}
$$

303 304

\section{Wall Shear Stress Gradient}

ECs which line the artery are often denuded by the stenting procedure and must be replaced to prevent IH. ${ }^{7,41}$ However, in vitro studies show that bovine ECs migrate away from areas of high WSSG with magnitudes above $5000 \mathrm{~N} / \mathrm{m}^{39}$ and $3400 \mathrm{~N} / \mathrm{m}^{3}$. ${ }^{42}$ As such, there are likely to be less ECs in areas of high WSSG in the stented artery. In computational studies, sites that are susceptible to IH have been correlated with sites where the WSSG has been predicted to exceed $200 \mathrm{~N} / \mathrm{m}^{3}$ in an end to side anastomosis model ${ }^{20,34}$ and a rabbit iliac model. $^{26}$

The WSSG is a measure of the spatial rate of change of the WSS vector and in a local coordinate system is calculated as

$$
\mathrm{WSSG}=\left[\left(\frac{\partial \tau_{w, m}}{\partial m}\right)^{2}+\left(\frac{\partial \tau_{w, n}}{\partial n}\right)^{2}\right]^{1 / 2}
$$

where $m$ is the WSS direction and $n$ is the direction tangential to the arterial surface and normal to $m$. The WSSG is calculated locally at each node using least squares fitting with singular value decomposition to find the "best fit" for the gradient components from the data at the surrounding nodes.

\section{Wall Shear Stress Angle Gradient}

ECs align with the flow direction creating a selective barrier to blood borne particles such as inflammatory cells. $^{29}$ Sudden directional changes in the WSS may lead to abnormal stresses on the junctions between these barrier cells resulting in increased permeability and risk of inflammation, a precursor of $\mathrm{IH}^{44}$ The WSSAG vector has been suggested as a meshindependent variable to quantify these directional changes. $^{28}$
The magnitude of the WSSAG is calculated as

$$
\mathrm{WSSAG}=\left(\left(\frac{\partial \phi}{\partial m}\right)^{2}+\left(\frac{\partial \phi}{\partial n}\right)^{2}\right)^{1 / 2}
$$

where $\phi$ is the angular difference between the timeaveraged WSS vector at the node of interest $\vec{\tau}_{0}$ and the corresponding vector at the neighbor node $\vec{\tau}_{\mathrm{r}}$ and is computed as

$$
\phi= \pm \cos ^{-1}\left(\frac{\vec{\tau}_{0} \cdot \vec{\tau}_{\mathrm{r}}}{\left|\vec{\tau}_{0}\right|\left|\vec{\tau}_{\mathrm{r}}\right|}\right), \quad-\pi<\phi \leq \pi
$$

for each of the neighbor nodes. At the node of interest the value of $\phi$ is set to zero and the WSSAG is calculated using a similar method to the WSSG calculation. The WSSAG thus calculated is mesh dependent only at points of boundary layer separation and reattachment in the flow, with values tending toward infinity as the mesh spacing reduces to zero. To prevent these flow features from affecting the WSSAG, an upper limit of $300 \mathrm{rad} / \mathrm{mm}$ is set on the variable. This approximately corresponds to the WSSAG magnitude created by the maximum angular difference between two WSS vectors $(\pi)$ acting on two small $(\sim 10 \mu \mathrm{m})$ adjacent ECs.

\section{Oscillatory Shear Index}

Time-dependent directional changes in the WSS may also lead to endothelial dysfunction. Specifically, areas of oscillating WSS have been shown to correlate with atherosclerotic plaque location. ${ }^{19}$ Transient directional changes in WSS are quantified by the oscillatory shear index (OSI). It is possible that areas of high OSI in the stented artery increase the risk of IH through endothelial dysfunction.

The OSI is calculated as

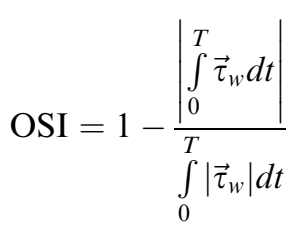

where $T$ is the period of the cardiac cycle. The range of this variable is from 0 for unidirectional flow, to a maximum of 1 in areas of highly-altered fullyoscillatory WSS.

\section{Analysis of Results}

The WSS, WSSG, WSSAG and OSI variables, initially calculated at the element vertices, are finally faceaveraged for analysis. The area distribution of these face-averaged variables is visualized using histograms by displaying the amount of area contained between 
specific intervals of the variable value. In addition to this qualitative technique, the area-averaged mean, standard deviation, and kurtosis of the distribution of each variable are also calculated for quantitative analysis. The area-averaged mean is calculated as

$$
\mu=\frac{\sum_{j=1}^{e}\left(A_{j} \times \phi_{j}\right)}{\sum_{j=1}^{e} A_{j}}
$$

where $\phi_{j}$ is the face-averaged variable value at the face $j, A_{j}$ is the surface area of the face $j$ and the summation is over e mesh faces. A high mean is desirable for the WSS, whereas a low mean is desirable for the WSSG, WSSAG and OSI. The area-averaged standard deviation of the distribution is calculated as

$$
\sigma=\sqrt{\frac{\sum_{j=1}^{e}\left[\left(A_{j}\right) \times\left(\phi_{j}-\mu\right)^{2}\right]}{\sum_{j=1}^{e} A_{j}}}
$$

where the terms are as before. The standard deviation provides a measure of the typical difference between variable values and the mean value of the distribution. Low values of standard deviation signify that the mean is a better representation of values everywhere on the artery wall. However, the standard deviation can be heavily influenced by extreme variable values far from the mean. The kurtosis, calculated as

$$
K=\frac{\sum_{j=1}^{e}\left[\left(A_{j}\right) \times\left(\phi_{j}-\mu\right)^{4}\right]}{\sum_{j=1}^{e}\left(A_{j} \times \sigma^{4}\right)}
$$

provides a measure of how heavily influenced the standard deviation is by extreme values in the distribution. A mean value may still be a good representation of the variable values everywhere in the artery even though the standard deviation is high, as long as the kurtosis is also high.

For each variable, the distributions associated with each stent are compared using Cohen's $d$ which measures the standardized difference between the magnitudes of the distributions. Comparing stent A to stent $\mathrm{B}$, Cohen's $d$ is calculated as

$$
d=\frac{\mu_{\text {stent B }}-\mu_{\text {stent A }}}{\sigma_{\text {pooled }}}
$$

where

$$
\sigma_{\text {pooled }}=\sqrt{\left(\frac{\sigma_{\text {stent A }}+\sigma_{\text {stent B }}}{2}\right)^{2}}
$$

For WSS, negative $d$ values are produced if stent A is the better stent and conversely for WSSG, WSSAG and OSI, positive $d$ values favor stent $\mathrm{A}$. The value of $d$ indicates the difference between the performances of the stents with regard to each variable.

\section{RESULTS}

Results are displayed in histograms for all four WSS-based variables. Logarithmic scales are used where appropriate to display all the relevant information. The area in the histograms is normalized by the total area analyzed which is the tissue area confined within the axial limits of the stent. Contour plots of the WSS, WSSG, WSSAG and OSI are presented in Fig. 4 for visualization. Illustrations of WSS vectors and the computational mesh near a Bx strut are shown in Figs. $5 \mathrm{a}$ and $5 \mathrm{~b}$, respectively.

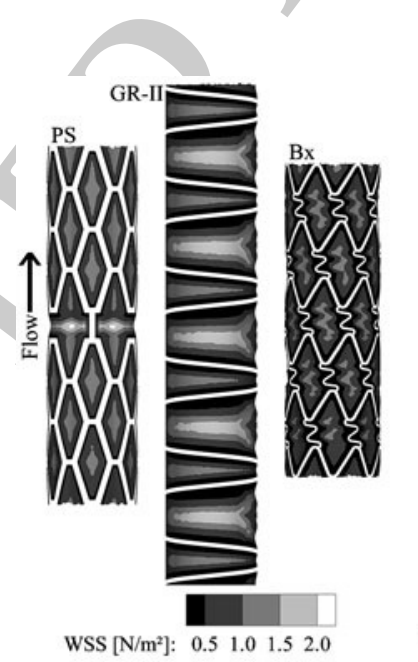

WSS $\left[\mathrm{N} / \mathrm{m}^{2}\right]: \begin{array}{lllll}0.5 & 1.0 & 1.5 & 2.0\end{array}$

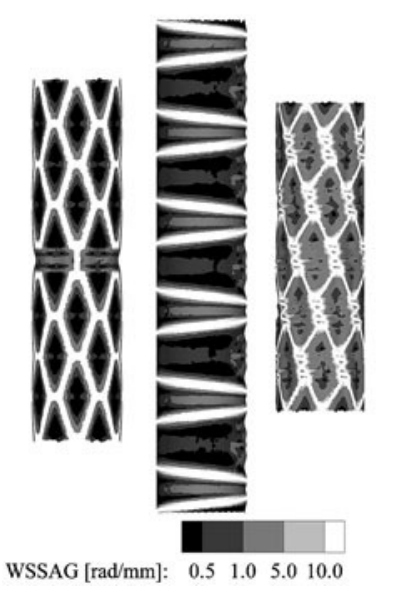

WSSG [N/m']: $\begin{array}{llll}500 & 1000 & 1500 & 2000\end{array}$

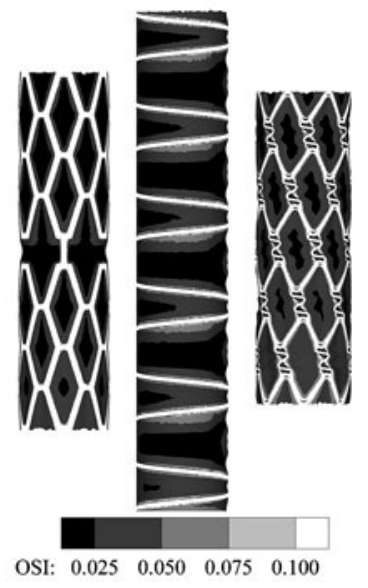

FIGURE 4. Contour plots of the WSS, WSSG, WSSAG and OSI predicted on the artery wall after implantation of the coronary stents. 
(a)

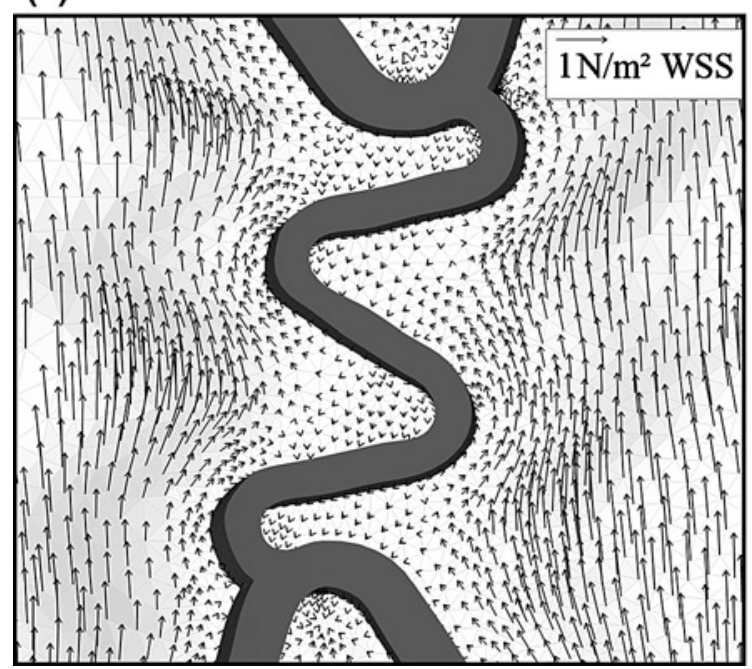

(b)

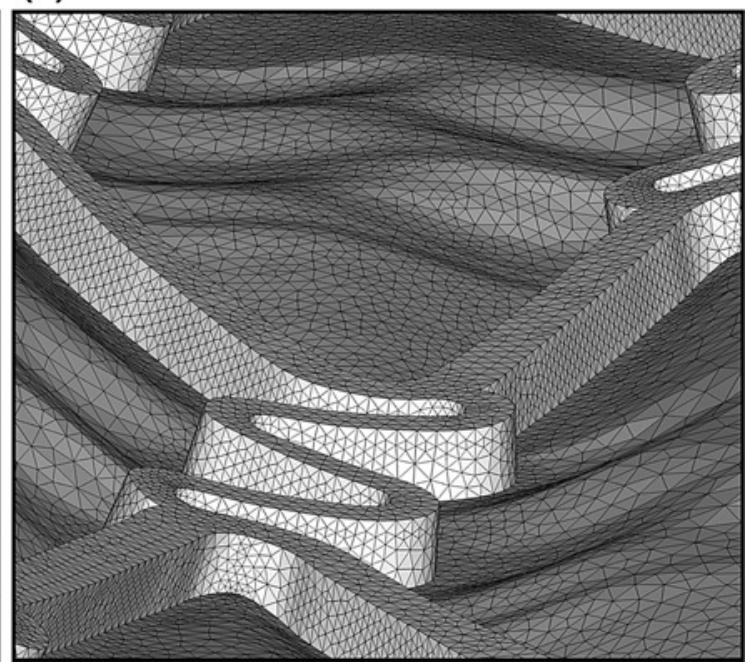

FIGURE 5. (a) WSS vectors in the vicinity of an S-connector of the Bx stent and (b) a view of the surface mesh of the uneven prolapse tissue around the same S-connector.

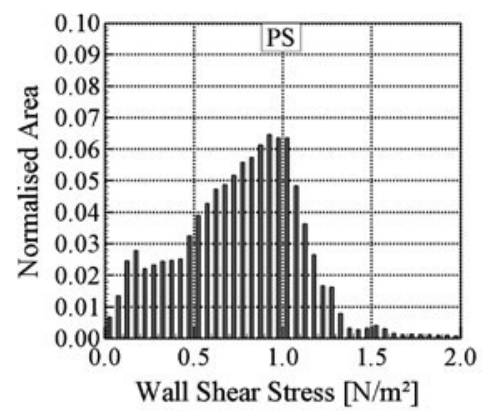

Stent

Palmaz Schatz

Giantirco Roubin II

Bx-Velocity
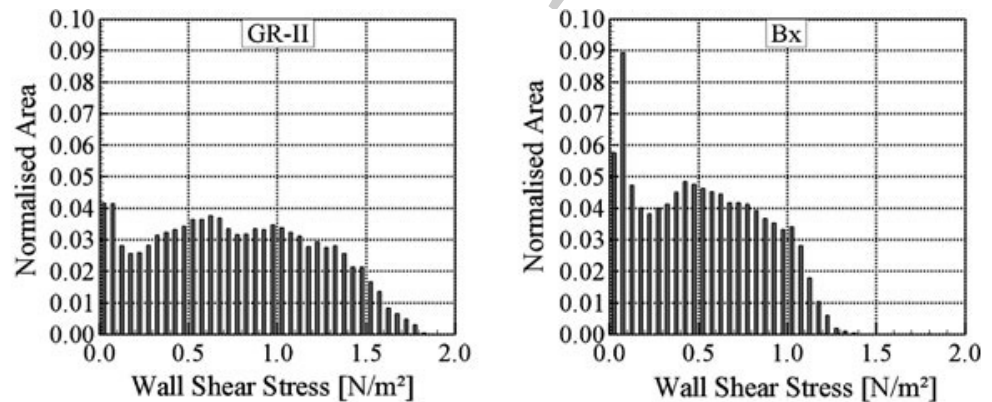

Mean $\left[\mathrm{N} / \mathrm{m}^{2}\right] \quad$ Standard Deviation $\left[\mathrm{N} / \mathrm{m}^{2}\right]$ Kurtosis

$\begin{array}{lll}0.760 & 0.341 & 3.262 \\ 0.764 & 0.454 & 1.999 \\ 0.522 & 0.336 & 1.925\end{array}$

FIGURE 6. Distributions of the WSS. The bars represent the amount of normalized area with WSS values bounded by the tick marks on the abscissa. Bin widths are $0.05 \mathrm{~N} / \mathrm{m}^{2}$.

\section{Wall Shear Stress}

The distribution of the WSS is presented in Fig. 6 for the three stents. Mean WSS values are similar for the PS and GR-II stents with values of 0.760 and $0.764 \mathrm{~N} / \mathrm{m}^{2}$, respectively. The mean is substantially lower for the Bx stent with a value of $0.522 \mathrm{~N} / \mathrm{m}^{2}$. These WSS values are $25-50 \%$ lower than those expected for an unstented $3.2 \mathrm{~mm}$ artery under similar flow conditions $\left(-1.0 \mathrm{~N} / \mathrm{m}^{2}\right)$. The results therefore predict that insertion of these stents reduces the WSS on the artery wall. Figure 4 shows that WSS values are reduced below $1.0 \mathrm{~N} / \mathrm{m}^{2}$ in large areas around all stent struts. This is a similar result to previous studies. ${ }^{23,24}$ The standard deviation of the WSS is slightly higher for the GR-II stent, and the relatively low kurtosis reveals this is not due to extreme values but rather a wider spread of WSS values in the artery. The $63 \%$ higher value of kurtosis for the PS stent compared to the GR-II stent indicates that the PS mean WSS value represents a larger portion of the artery compared to the similar GR-II mean value. As such, conditions are more favorable in the artery implanted with the PS stent compared to the GR-II stent, with the worst WSS values created by implantation of the Bx stent. Large areas of low WSS are visible on the proximal side of the GR-II struts in Fig. 4, due to low flow velocity in this region. The thicker struts of the Bx stent have a notable effect on the near-strut low WSS region which 
is considerably larger than that for the similarly shaped PS stent. Comparing the stents gives PS to $\mathrm{Bx}$ $(d=-0.703)$, PS to GR-II $(d=-0.010)$ and GR-II to $\mathrm{Bx}(d=-0.613)$. This puts the stents in order from best to worst as PS, GR-II and Bx.

The commonly used threshold method of analysis shows that the PS stent has $22.4 \%$ of arterial tissue exposed to WSS $<0.5 \mathrm{~N} / \mathrm{m}^{2}$ compared with $32.3 \%$ for the GR-II stent and $49.5 \%$ for the $\mathrm{Bx}$ stent. In this case, through comparison with the proposed methodology, the threshold method is capable of identifying the alterations to the magnitude of the WSS after implantation of these particular stents.

\section{Wall Shear Stress Gradient}

The distribution of the WSSG is shown in Fig. 7. The GR-II stent has the highest mean value, followed by the $\mathrm{Bx}$ stent and finally the PS stent. Further examination of the results reveals the highest standard deviation and lowest kurtosis for the GR-II stent indicating a wider spread of WSSG values leading to more area subjected to higher magnitudes of WSSG. Comparing the PS and Bx stents, the distributions of WSSG in the artery are similar. The slightly lower mean and higher kurtosis marginally favors the PS as the stent with the best WSSG conditions. Figure 4 reveals the areas of highest WSSG at the articulation site and also proximal and distal to the struts that traverse the flow with the PS stent. High values of WSSG $\left(>2000 \mathrm{~N} / \mathrm{m}^{3}\right)$ are visible along the length of the GR-II stent. The high WSSG values are located on the surfaces of the unsupported tissue that is prolapsing into the artery. Uneven prolapse near the
S-connectors of the Bx stent has also produced high WSSG values. Comparing the stents gives PS to $\mathrm{Bx}$ $(d=0.159)$, PS to GR-II $(d=0.837)$ and GR-II to $\mathrm{Bx}$ $(d=-0.764)$. This puts the stents in order from best to worst as PS, Bx and GR-II. Comparing the stents using the threshold method of analysis, the PS stent exposes $97.6 \%$ of the arterial tissue to WSSG > $200 \mathrm{~N} / \mathrm{m}^{3}$ compared with $97.4 \%$ with the GR-II stent and $98.9 \%$ with the $\mathrm{Bx}$ stent. These results are very similar, and demonstrate that when analyzing the WSSG, the threshold method is unable to distinguish between the stents.

\section{Wall Shear Stress Angle Gradient}

The distribution of the WSSAG is presented in Fig. 8 using both semi-log and $\log -\log$ plots to ensure firstly, that the trend of the data is identifiable and secondly, that all of the analyzed area is visible on the plot. The mean value is highest for the $\mathrm{Bx}$ stent indicating that implantation of this stent leads to the greatest alteration to the WSS direction. This is followed by the GR-II stent which produces the highest standard deviation and lowest kurtosis. The PS has the best result with a mean WSSAG value of $2.405 \mathrm{rad} /$ $\mathrm{mm}$. The log-log histograms in Fig. 8 show that all stents have a small amount of area (approximately $0.03 \%$ ) in the $100-200 \mathrm{rad} / \mathrm{mm}$ histogram range and the GR-II and Bx have very small amounts of area (approximately $0.001 \%$ ) in the $200-300 \mathrm{rad} / \mathrm{mm}$ range. Figure 4 shows the areas with the highest values of WSSAG located around the S-connectors of the $\mathrm{Bx}$ stent and also proximal and distal to the GR-II stent struts which traverse the flow. Large areas of low
490

491

492

493

494

495

496

497

498

499

500

501

502
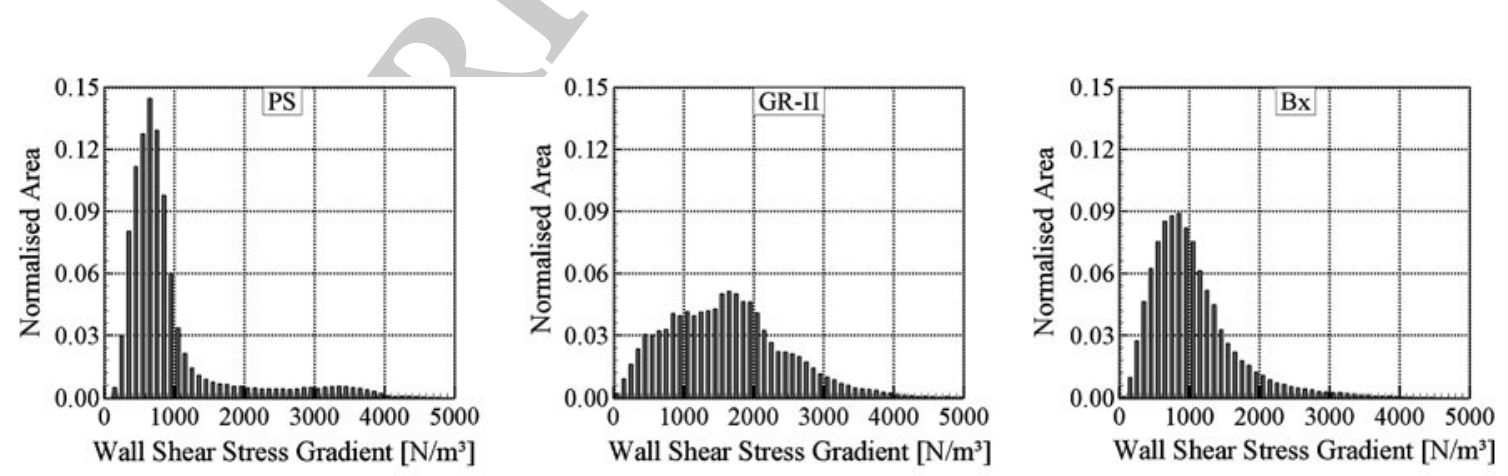

$\begin{array}{lccc}\text { Stent } & \text { Mean }\left[\mathrm{N} / \mathrm{m}^{3}\right] & \text { Standard Deviation }\left[\mathrm{N} / \mathrm{m}^{3}\right] & \text { Kurtosis } \\ \text { Palmaz Schatz } & 938.8 & 783.3 & 8.9 \\ \text { Giantirco Roubin II } & 1626.9 & 858.4 & 3.9 \\ \text { Bx-Velocity } & 1051.6 & 631.4 & 8.0\end{array}$

FIGURE 7. Distributions of the WSSG. The bars represent the amount of normalized area with WSSG values bounded by the tick marks on the abscissa. Bin widths are $100 \mathrm{~N} / \mathrm{m}^{3}$. 

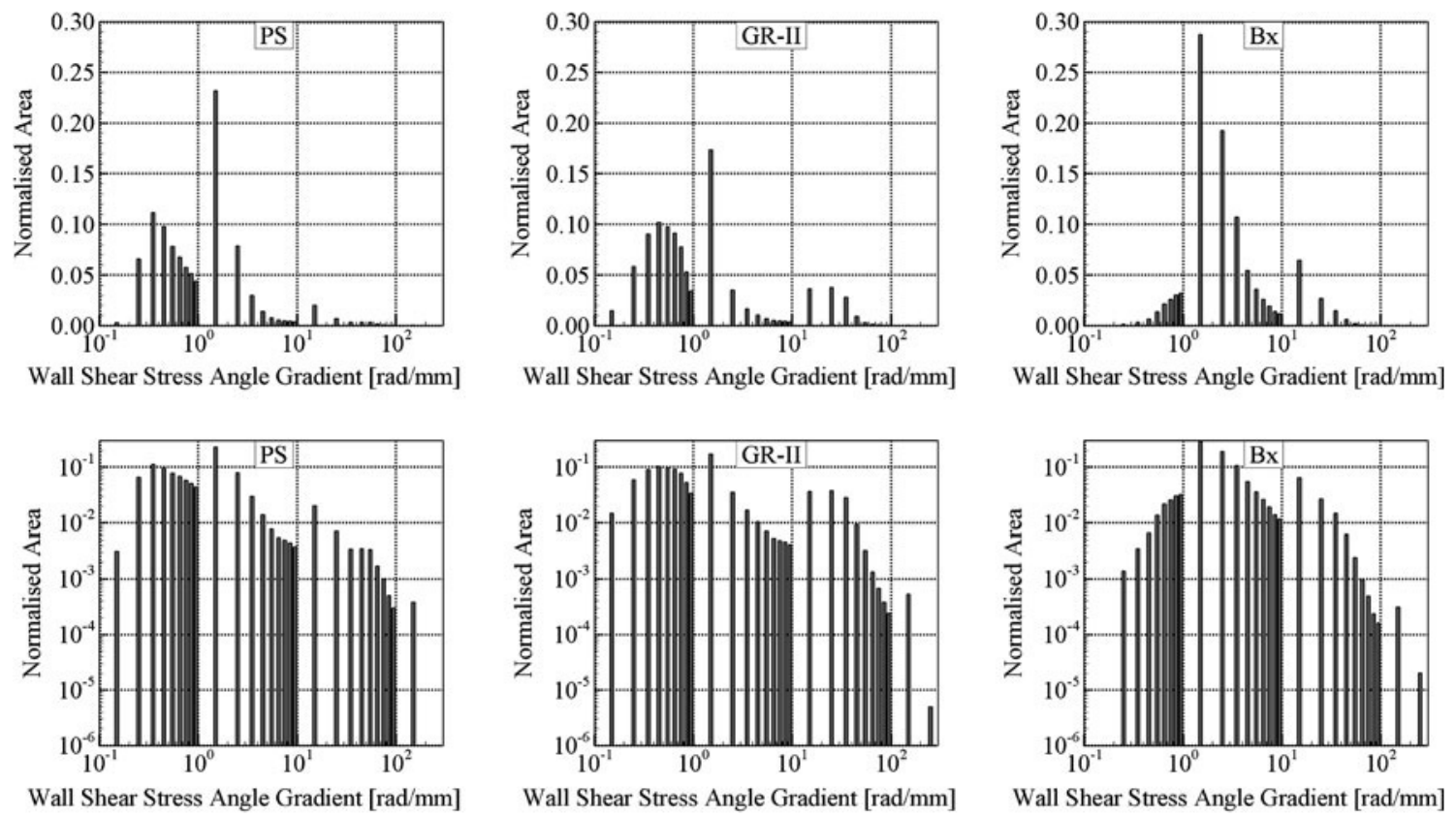

$\begin{array}{lccc}\text { Stent } & \text { Mean [rad/mm] } & \text { Standard Deviation [rad/mm] } & \text { Kurtosis } \\ \text { Palmaz Schatz } & 2.405 & 7.176 & 82.818 \\ \text { Giantirco Roubin II } & 4.260 & 10.041 & 27.853 \\ \text { Bx-Velocity } & 5.009 & 8.199 & 52.332\end{array}$

FIGURE 8. Distributions of the WSSAG. The bars represent the amount of normalized area with WSSAG values bounded by the tick marks on the abscissa. Bin widths are distributed logarithmically. Additional log-log plots are provided to display all of the arterial area analyzed.

WSSAG are evident in between the struts of the PS stent with values peaking near the small portions of the struts that traverse the flow. Comparing the stents gives PS to $\mathrm{Bx}(d=0.338)$, PS to GR-II $(d=0.213)$ and GR-II to $\mathrm{Bx}(d=0.082)$. This puts the stents in order from best to worst as PS, GR-II and Bx.

This variable provides a new perspective on the alterations to the WSS induced by stent implantation that is not commonly analyzed. Therefore, no threshold value exists in the literature to allow for comparison of techniques.

\section{Oscillatory Shear Index}

Implantation of the Bx stent creates the greatest transient variation in the WSS direction as the mean of the OSI is 30 and $54 \%$ higher than for the GR-II and PS stents, respectively. As shown in Fig. 9, the Bx stent also has the highest standard deviation and lowest kurtosis of the three stents indicating that this stent performs the worst with regard to OSI. Comparatively the PS stent has the lowest mean and standard deviation with the highest kurtosis indicating that implantation of this stent creates the least alteration to the transient WSS direction. Since the range of the OSI is from 0 to 1 , the mean values of OSI in the arteries for the three stents are very low and as such, the differences in the distributions may be viewed as insignificant. However, the OSI quantifies transient directional changes in flow direction which may only be significant for IH in specific small areas of the artery which could amplify the significance of these small differences. The $\log -\log$ plot shows small areas in the 0.4-0.5 range of OSI for the PS stent and small areas in the 0.5-0.6 range for the GR-II and Bx stents. This indicates regions where there are highly transient directional changes in the WSS vector. Since the inlet flow is unidirectional, Fig. 4 shows that most of the stented artery has low values $(<0.05)$ of OSI. The OSI is slightly elevated in the proximal region for all stents and has its peak values very close to the GR-II stent wires and in select locations near the Bx struts and S-connectors as shown in Fig. 4. Comparing the stents gives PS to $\mathrm{Bx}(d=0.620)$, PS to GR-II $(d=0.315)$ and GR-II to $\mathrm{Bx}(d=0.380)$. This puts the stents in order from best to worst as PS, GR-II and Bx. 

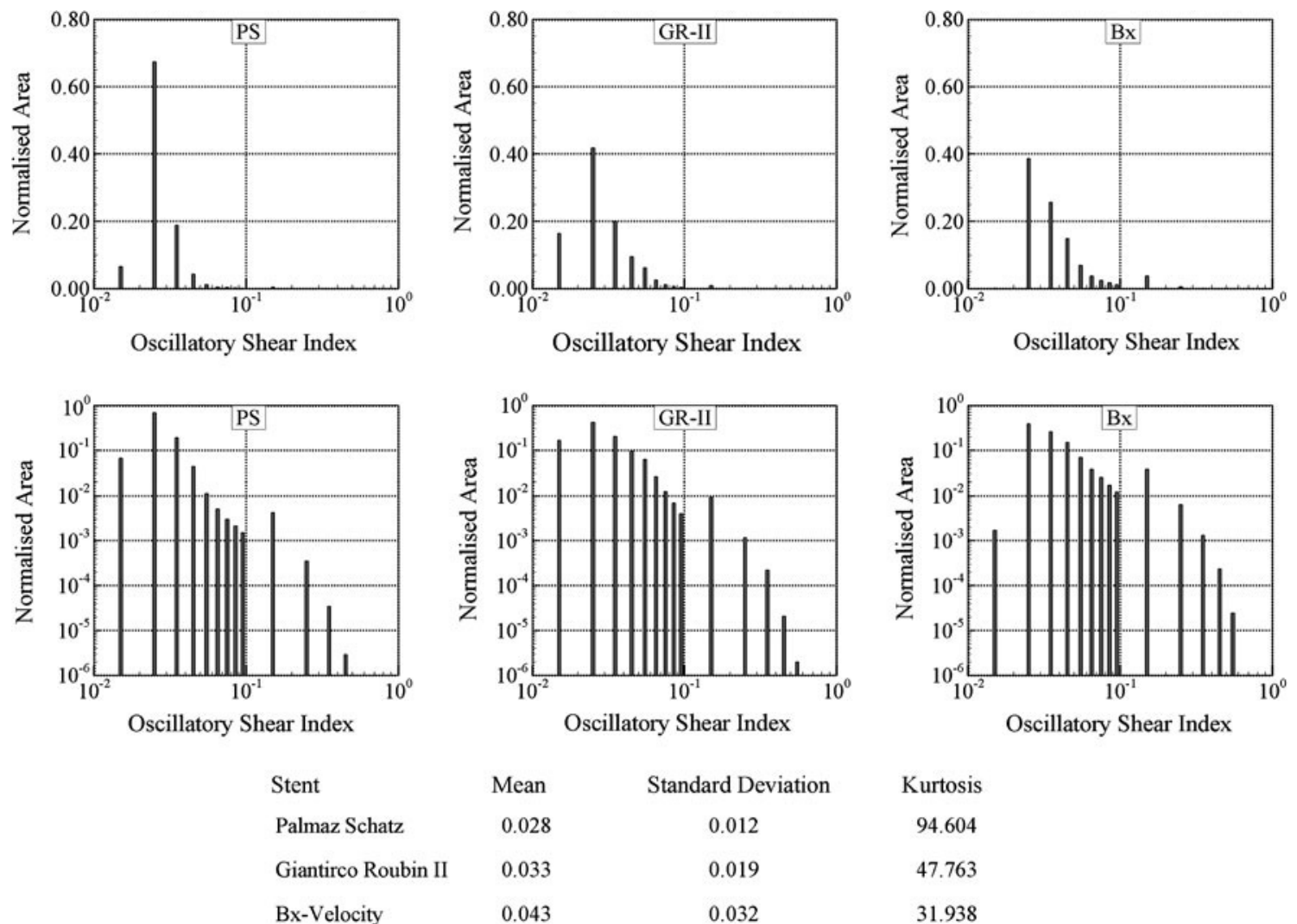

Mean
0.028
0.033
0.043

Standard Deviation
0.012
0.019
0.032

Kurtosis
94.604
47.763
31.938

FIGURE 9. Distributions of the OSI. The bars represent the amount of normalized area with OSI values bounded by the tick marks on the abscissa. Bin widths are distributed logarithmically. Additional log-log plots are provided to display all of the arterial area analyzed.

\section{DISCUSSION}

A new methodology is proposed to fully elucidate the alterations in arterial WSS induced by stent deployment. Four variables are employed in the methodology, each of which highlights a different type of alteration of the arterial WSS which could lead to IH development. The proposed method of analyzing the WSS-based variables provides a clear qualitative and quantitative assessment of each variable distribution making it possible to accurately assess the hemodynamic performance of a stent.

When the methodology is applied to the three stents, the results have favored the PS as the implanted stent which creates the least alteration to the WSS in the artery. The WSS, WSSG and WSSAG variables rank the PS stent the highest. The OSI also favors the PS stent; however the magnitudes of the mean OSI values are quite small for the stents. Nevertheless, the histograms and contour plots do show that the GR-II and $\mathrm{Bx}$ stents create higher magnitudes of OSI around the stent struts compared to the PS stent. The WSS, WSSAG and OSI variables rank the GR-II ahead of the Bx stent, with the only exception to the trend being the WSSG where the GR-II ranks the lowest. Overall, the methodology indicates the stents hemodynamically perform in the order of PS, GR-II and finally Bx.

Using the threshold method, the WSS variable identifies the PS as the best stent, followed by the GR-II and the Bx stents whereas the WSSG variable yields inconclusive results. In this case, the threshold method has managed to sufficiently distinguish between the stents using one variable, and also rank them in agreement with the proposed methodology. However, the threshold method does not quantify the complex hemodynamic disturbances that are identified in this paper. Furthermore, the threshold method has been proven unable to distinguish between stents in previous studies, ${ }^{3,11,23}$ where the proposed method may have proved more successful. An example of complex stent induced hemodynamic disturbance is shown in Fig. 5a where WSS vectors are shown to quickly change magnitude and direction due to the uneven prolapse shown in Fig. 5b. This leads to high WSSG and WSSAG in this area which may well lead to increased IH. The OSI in this area is also shown to be high. These effects are not identified by the use of the threshold method and such features could easily have been overlooked in the previous studies. 
TABLE 2. Angiographic restenosis rates for the PS, GR-II and Bx stents from five clinical trials.

\begin{tabular}{|c|c|c|c|c|}
\hline Trial (year) & Trial design & No. of patients & Follow up (months) & Restenosis rate $(\%)$ \\
\hline Lanskey et al. ${ }^{27}$ (2000) & GR-II vs. PS & 755 & 12 & 47.3 vs. 20.6 \\
\hline NIRVANA $^{2}(2001)$ & NIR vs. PS & 849 & 9 & 19.3 vs. 22.4 \\
\hline ASCENT $^{1}(2001)$ & ML vs. PS & 529 & 9 & 16.0 vs. 22.1 \\
\hline RAVEL DES ${ }^{40}(2002)$ & SES vs. Bx & 283 & 6 & 0.0 vs. 23.4 \\
\hline ISAR-STERO II ${ }^{35}$ (2003) & ML vs. Bx & 611 & 6 & 17.9 vs. 31.4 \\
\hline
\end{tabular}

PS Palmaz Schatz (Cordis, Johnson and Johnson, NJ, USA), NIR NIR stent (Boston Scientific, MA, USA), ML Multilink (Guidant, CA, USA), SES Sirolimus-eluting stent.

614 The clinical performance of these stents is available 615 from the results of several clinical trials shown in 616 Table 2. The most commonly used method of com617 parison of BMSs is angiographic restenosis rates, 618 defined as percentage of patients with $>50 \%$ renar619 rowing of the target vessel at follow up. The PS and 620 GR-II stents were directly compared in a trial con621 sisting of 755 patients with de novo lesions. ${ }^{27}$ Reste622 nosis rates were found to be statistically significant $623(p<0.001)$ between the two stents with values of 47.3 624 and $20.6 \%$ for the GR-II and PS, respectively. A 625 possible factor in the poor GR-II result is the "clam626 shell" deployment which is likely to cause more arterial 627 damage than for the slotted-tube type stents such as 628 the PS. "Infrequent optimal GR-II size selection" was 629 also noted in the study which would likely contribute 630 to poor stent performance. The PS stent has also been 631 involved in the stent equivalency trials ASCENT $^{1}$ and 632 NIRVANA ${ }^{2}$ and had restenosis rates of 22.1 and $63322.4 \%$, respectively. There were similar criteria for 634 inclusion in these three trials such as native vessel 635 diameter of greater than $3 \mathrm{~mm}$, de novo lesions, and 636 similar study end points. The Bx stent had a restenosis 637 rate of $31.4 \%$ in the ISAR-STEREO-II ${ }^{35}$ which had 638 patients with de novo and restenotic lesions, but sim639 ilar vessel diameter and trial end points. The Bx stent 640 also had a restenosis rate of $23.4 \%$ in the control arm 641 of the RAVAL DES trial ${ }^{40}$ where inclusion criteria 642 were a de novo lesion with native vessel diameter 643 between 2.5 and $3.5 \mathrm{~mm}$. While one must be cautious 644 when comparing the results of different stent trials, it 645 would appear that the PS stent has the best in vivo 646 results, followed in turn by the Bx and GR-II stents. 647 From the results of the current study it would appear 648 that hemodynamics are influential in the restenosis 649 rates associated with the PS and Bx stents. From the 650 CFD results, the GR-II stent performed worse than the 651 PS stent hemodynamically. However, it would have to 652 be concluded that other influential factors were also to 653 blame for the stents poor clinical performance as it had 654 such a severe restenosis rate.

655 Limitations to the methodology employed in this 656 paper include the assumptions of fully-developed laminar flow, a rigid stent and arterial wall, and the omission of a stenotic plaque. Whilst the depth of tissue prolapse is based on FEA data ${ }^{36}$ the shape of the protruding tissue is a further limitation as it is idealized and based entirely on the geometry of the stent. Curvature and taper of the artery have also been omitted in the analysis for simplicity. The outlet boundary condition of a fixed pressure is a limitation creating a non-physiological transient pressure in the CFD model. However, this outlet boundary condition is the standard practice for modeling of pulsatile flow in arteries. ${ }^{3-5,13,21,23,25,37}$ With this boundary condition, the CFD software calculates the necessary inlet pressure to drive the flow. The velocity which is specified at the inlet should therefore maintain reasonable physiological accuracy in the computational domain.

The objective of this work is to introduce a more complete method of analyzing the alterations to WSS acting on the living tissue in the stented artery. As such, this method of stent assessment should assist in stent design in the future and is applicable to bare metal, drug-eluting and any future stents that alter the WSS in the artery after implantation.

\section{ACKNOWLEDGMENTS}

This paper is the result of 4 years of effort and was supported by the Department of Mechanical Engineering in the Dublin Institute of Technology (DIT) and also the Irish Research Council for Science Engineering and Technology (IRCSET).

\section{REFERENCES}

${ }^{1}$ Baim, D. S., D. E. Cutlip, M. Midei, T. J. Linnemeier, T. Schreiber, D. Cox, D. Kereiakes, J. J. Popma, L. Robertson, R. Prince, A. J. Lansky, K. K. L. Ho, and R. E. Kuntz. Final results of a randomized trial comparing the MULTI-LINK stent with the Palmaz-Schatz stent for narrowings in native coronary arteries. Am. J. Cardiol. $87: 157-162,2001$. 
${ }^{2}$ Baim, D. S., D. E. Cutlip, C. D. O'Shaughnessy, J. B. Hermiller, D. J. Kereiakes, A. Giambartolomei, S. Katz, A. J. Lansky, M. Fitzpatrick, J. J. Popma, K. K. L. Ho, M. B. Leon, and R. E. Kuntz. Final results of a randomized trial comparing the NIR stent to the PalmazSchatz stent for narrowings in native coronary arteries. Am. J. Cardiol. 87:152-156, 2001.

${ }^{3}$ Balossino, R., F. Gervaso, F. Migliavacca, and G. Dubini. Effects of different stent designs on local hemodynamics in stented arteries. J. Biomech. 41:1053-1061, 2008.

${ }^{4}$ Banerjee, R. K., S. B. Devarakonda, D. Rajamohan, and L. H. Back. Developed pulsatile flow in a deployed coronary stent. Biorheology 44:91-102, 2007.

${ }^{5}$ Berry, J., A. Santamarina, J. E. Moore, Jr, S. Roychowdhury, and W. Routh. Experimental and computational flow evaluation of coronary stents. Ann. Biomed. Eng. 28:386-398, 2000.

${ }^{6}$ Chen, H. Y., J. Hermiller, A. K. Sinha, M. Sturek, L. Zhu, and G. S. Kassab. Effects of stent sizing on endothelial and vessel wall stress: potential mechanisms for in-stent restenosis. J. Appl. Physiol. 106:1686-1691, 2009.

${ }^{7}$ Clowes, A. W., and M. M. Clowes. Kinetics of cellular proliferation after arterial injury. IV. Heparin inhibits rat smooth muscle mitogenesis and migration. Circ. Res. 58:839-845, 1986.

${ }^{8}$ Colombo, A., J. Drzewiecki, A. Banning, E. Grube, K. Hauptmann, S. Silber, D. Dudek, S. Fort, F. Schiele, K. Zmudka, G. Guagliumi, and M. E. Russell. Randomized study to assess the effectiveness of slow- and moderate-release polymer-based paclitaxel-eluting stents for coronary artery lesions. Circulation 108:788-794, 2003.

${ }^{9}$ DePaola, N., M. A. J. Gimbrone, P. F. Davies, and C. F. Dewey. Vascular endothelium responds to fluid shear stress gradients. Arterioscler. Thromb. 12:1254-1257, 1992.

${ }^{10}$ Duraiswamy, N., J. M. Cesar, R. T. Schoephoerster, and J. E. Moore, Jr. Effects of stent geometry on local flow dynamics and resulting platelet deposition in an in vitro model. Biorheology 45:547-561, 2008.

${ }^{11}$ Duraiswamy, N., R. T. Schoephoerster, and J. E. Moore, Jr. Comparison of near-wall hemodynamic parameters in stented artery models. J. Biomech. Eng. 131:061006, 2009.

${ }^{12}$ Escaned, J., J. Goicolea, F. Alfonso, M. J. Perez, R. Hernandez, A. Fernandez, C. Banuelos, and C. Macaya. Propensity and mechanisms of restenosis in different coronary stent designs: complementary value of the analysis of the luminal gain-loss relationship. J. Am. Coll. Cardiol. 34: 1490-1497, 1999.

${ }^{13}$ Frank, A. O., P. W. Walsh, and J. E. Moore, Jr. Computational fluid dynamics and stent design. Artif. Organs 26: 614-621, 2002.

${ }^{14}$ García, J., A. Crespo, J. Goicolea, M. Sanmartín, and C. García. Study of the evolution of the shear stress on the restenosis after coronary angioplasty. J. Biomech. 39:799-805, 2006.

${ }^{15} \mathrm{He}$, Y., N. Duraiswamy, A. O. Frank, and J. E. Moore, Jr. Blood flow in stented arteries: a parametric comparison of strut design patterns in three dimensions. J. Biomech. Eng. 127:637-647, 2005.

${ }^{16}$ Kastrati, A., J. Mehilli, J. Dirschinger, F. Dotzer, H. Schuhlen, F. J. Neumann, M. Fleckenstein, C. Pfafferott, M. Seyfarth, and A. Schomig. Intracoronary stenting and angiographic results: strut thickness effect on restenosis outcome (ISAR-STEREO) trial. Circulation 103:28162821, 2001.
${ }^{17}$ Kastrati, A., J. Mehilli, J. Dirschinger, J. Pache, K. Ulm, H. Schuhlen, M. Seyfarth, C. Schmitt, R. Blasini, F. J. Neumann, and A. Schomig. Restenosis after coronary placement of various stent types. Am. J. Cardiol. 87:34-39, 2001.

${ }^{18} \mathrm{Ku}$, D. N. Blood flow in arteries. Аnпи. Rev. Fluid Mech. 29:399-434, 1997.

${ }^{19} \mathrm{Ku}$, D. N., D. P. Giddens, C. K. Zarins, and S. Glagov. Pulsatile flow and atherosclerosis in the human carotid bifurcation. Positive correlation between plaque location and low oscillating shear stress. Arteriosclerosis 5:293-302, 1985.

${ }^{20}$ Kute, S. M., and D. A. Vorp. The effect of proximal artery flow on the hemodynamics at the distal anastomosis of a vascular bypass graft: computational study. J. Biomech. Eng. 123:277-283, 2001.

${ }^{21}$ LaDisa, J., L. Olson, H. Douglas, D. Warltier, J. Kersten, and P. Pagel. Alterations in regional vascular geometry produced by theoretical stent implantation influence distributions of wall shear stress: analysis of a curved coronary artery using 3D computational fluid dynamics modeling. Biomed. Eng. Online 5:40-46, 2006.

${ }^{22}$ LaDisa, J. F., I. Guler, L. E. Olson, D. A. Hettrick, J. R. Kersten, D. C. Warltier, and P. S. Pagel. Threedimensional computational fluid dynamics modeling of alterations in coronary wall shear stress produced by stent implantation. Ann. Biomed. Eng. 31:972-980, 2003.

${ }^{23}$ LaDisa, J. F., L. Olson, D. Hettrick, D. Warltier, J. Kersten, and P. Pagel. Axial stent strut angle influences wall shear stress after stent implantation: analysis using 3D computational fluid dynamics models of stent foreshortening. Biomed. Eng. Online 4:59-61, 2005.

${ }^{24}$ LaDisa, J. F., L. E. Olson, I. Guler, D. A. Hettrick, S. H. Audi, J. R. Kersten, D. C. Warltier, and P. S. Pagel. Stent design properties and deployment ratio influence indexes of wall shear stress: a three-dimensional computational fluid dynamics investigation within a normal artery. J. Appl. Physiol. 97:424-430, 2004.

${ }^{25}$ LaDisa, J. F., L. E. Olson, I. Guler, D. A. Hettrick, J. R. Kersten, D. C. Warltier, and P. S. Pagel. Circumferential vascular deformation after stent implantation alters wall shear stress evaluated with time-dependent 3D computational fluid dynamics models. J. Appl. Physiol. 98:947957, 2005

${ }^{26}$ LaDisa, J. F., L. E. Olson, R. C. Molthen, D. A. Hettrick, P. F. Pratt, M. D. Hardel, J. R. Kersten, D. C. Warltier, and P. S. Pagel. Alterations in wall shear stress predict sites of neointimal hyperplasia after stent implantation in rabbit iliac arteries. Am. J. Physiol. Heart Circ. Physiol. 288: H2465-H2475, 2005.

${ }^{27}$ Lansky, A. J., G. S. Roubin, C. D. O’Shaughnessy, P. B. Moore, L. S. Dean, A. E. Raizner, R. D. Safian, J. P. Zidar, J. L. Kerr, J. J. Popma, R. Mehran, R. E. Kuntz, and M. B. Leon. Randomized comparison of GR-II stent and Palmaz-Schatz stent for elective treatment of coronary stenoses. Circulation 102:1364-1368, 2000.

${ }^{28}$ Longest, P. W., and C. Kleinstreuer. Computational haemodynamics analysis and comparison study of arteriovenous grafts. J. Med. Eng. Technol. 24:102-110, 2000.

${ }^{29}$ Malek, A. M., S. L. Alper, and S. Izumo. Hemodynamic shear stress and its role in atherosclerosis. JAMA 282:2035-2042, 1999.

${ }^{30}$ Moore, J. E., and J. L. Berry. Fluid and solid mechanical implications of vascular stenting. Ann. Biomed. Eng. 30: 498-508, 2002. 
${ }^{31}$ Moses, J. W., M. B. Leon, J. J. Popma, P. J. Fitzgerald, D. R. Holmes, C. O'Shaughnessy, R. P. Caputo, D. J. Kereiakes, D. O. Williams, P. S. Teirstein, J. L. Jaegerand, and R. E. Kuntz. Sirolimus-eluting stents versus standard stents in patients with stenosis in a native coronary artery. N. Engl. J. Med. 349:1315-1323, 2003.

${ }^{32}$ Murphy, J., and F. Boyle. Assessment of the effects of increasing levels of physiological realism in the computational fluid dynamics analyses of implanted coronary stents. In: Proceedings of the Engineering in Medicine and Biology Society, 30th Annual International Conference of the IEEE, Vancouver, 2008, pp. 5906-5909.

${ }^{33}$ Nichols, W. W., and M. F. O'Rourke. The coronary circulation. In: McDonald's Blood Flow in Arteries Theoretical, Experimental and Clinical Principles, edited by J. Koster, S. Burrows, and N. Wilkinson. London: Hodder Arnold, 2005, pp. 326-327.

${ }^{34}$ Ojha, M. Spatial and temporal variations of wall shear stress within an end-to-side arterial anastomosis model. J. Biomech. 26:1377-1388, 1993.

${ }^{35}$ Pache, J. U., A. Kastrati, J. Mehilli, H. Schuhlen, F. Dotzer, J. O. Hausleiter, M. Fleckenstein, F. J. Neumann, U. Sattelberger, C. Schmitt, M. Muller, J. Dirschinger, and A. Schomig. Intracoronary stenting and angiographic results: strut thickness effect on restenosis outcome (ISARSTEREO-2) trial. J. Am. Coll. Cardiol. 41:1283-1288, 2003.

${ }^{36}$ Prendergast, P. J., C. Lally, S. Daly, A. J. Reid, T. C. Lee, D. Quinn, and F. Dolan. Analysis of prolapse in cardiovascular stents: a constitutive equation for vascular tissue and finite-element modelling. J. Biomech. Eng. 125:692699, 2003.

${ }^{37}$ Rajamohan, D., R. K. Banerjee, L. H. Back, A. A. Ibrahim, and M. A. Jog. Developing pulsatile flow in a deployed coronary stent. J. Biomech. Eng. 128:347-359, 2006.

${ }^{38}$ Rogers, C., and E. R. Edelman. Endovascular stent design dictates experimental restenosis and thrombosis. Circulation 91:2995-3001, 1995.

${ }^{39}$ Schwartz, R. S., K. C. Huber, J. G. Murphy, W. D. Edwards, A. R. Camrud, R. E. Vlietstra, and
D. R. Holmes. Restenosis and the proportional neointimal response to coronary artery injury: results in a porcine model. J. Am. Coll. Cardiol. 19:267-274, 1992.

${ }^{40}$ Serruys, P. W., M. Degertekin, K. Tanabe, A. Abizaid, J. E. Sousa, A. Colombo, G. Guagliumi, W. Wijns, W. K. Lindeboom, J. Ligthart, P. J. de Feyter, and M. Morice. Intravascular ultrasound findings in the multicenter, randomized, double-blind RAVEL (randomized study with the sirolimus-eluting velocity balloon-expandable stent in the treatment of patients with de novo native coronary artery lesions) trial. Circulation 106:798-803, 2002.

${ }^{41}$ Stemerman, M. B., T. H. Spaet, F. Pitlick, J. Cintron, I. Lejnieks, and M. L. Tiell. Intimal healing. The pattern of reendothelialization and intimal thickening. Am. J. Pathol. 87:125-142, 1977.

${ }^{42}$ Tardy, Y., N. Resnick, T. Nagel, M. A. Gimbrone, Jr., and C. F. Dewey, Jr. Shear stress gradients remodel endothelial monolayers in vitro via a cell proliferation-migration-loss cycle. Arterioscler. Thromb. Vasc. Biol. 17:3102-3106, 1997.

${ }^{43}$ Thury, A., J. J. Wentzel, R. V. H. Vinke, F. J. H. Gijsen, J. C. H. Schuurbiers, R. Krams, P. J. de Feyter, P. W. Serruys, and C. J. Slager. Focal in-stent restenosis near step-up: roles of low and oscillating shear stress. Circulation 105:185-187, 2002.

${ }^{44}$ Toutouzas, K., A. Colombo, and C. Stefanadis. Inflammation and restenosis after percutaneous coronary interventions. Eur. Heart J. 25:1679-1687, 2004.

${ }^{45}$ Vanhoutte, P. M. Endothelial dysfunction the first step toward coronary arteriosclerosis. Circ. J. 73:595-601, 2009. ${ }^{46}$ Wentzel, J. J., R. Krams, J. C. H. Schuurbiers, J. A. Oomen, J. Kloet, W. J. van der Giessen, P. W. Serruys, and C. J. Slager. Relationship between neointimal thickness and shear stress after wallstent implantation in human coronary arteries. Circulation 103:1740-1745, 2001.

${ }^{47}$ Zarins, C. K., D. P. Giddens, B. K. Bharadvaj, V. S. Sottiurai, R. F. Mabon, and S. Glagov. Carotid bifurcation atherosclerosis. Quantitative correlation of plaque localization with flow velocity profiles and wall shear stress. Circ. Res. 53:502-514, 1983.
866

867

868

869

870

871

872

873

874

875

876

877

878

879

880

881

882

883

884

885

886

887

888

889

890

891

892

893

894

895

896

897

898

899

900

901

902

903

904

905 